\title{
A Pragmatic Randomized Trial Comparing Telephone-Based Enhanced Pharmacy Care and Usual Care to Support Smoking Cessation
}

\author{
Jason Gong, MD; Christine L. Baker, JD, MPH; Kelly H. Zou, PhD; Marianna Bruno, PharmD, MPH; \\ Zhanna Jumadilova, MD, MBA; David Lawrence, PhD; Beth Wilson, MS, MPH; and Cynthia Ewel, PhD
}

\begin{abstract}
BACKGROUND: Smoking is the leading preventable cause of death, and tobacco control professionals continue to make progress in cessation efforts. Pharmacists can assist smokers seeking to quit by offering counseling on smoking cessation pharmacotherapies. Pragmatic randomized trials are useful for investigating practical questions about an intervention's risks, benefits, and costs in routine clinical practice.
\end{abstract}

OBJECTIVE: To evaluate an enhanced pharmacy care (EPC) program involving personalized pharmacist-provided telephone counseling for supporting prescription smoking cessation medications compared with usual care (UC).

METHODS: Cigarette smokers filling a newly prescribed smoking cessation pharmacotherapy and with pharmacy benefits managed by Express Scripts were recruited. Qualified subjects were randomized 1:1 to EPC and UC. Subjects in EPC received 3 telephone-counseling sessions from specialist pharmacists during the early course of the study, while subjects in UC did not receive any counseling sessions. Study outcomes were collected through telephone contact and using the Express Scripts prescription database. The primary outcome assessed the 1-week point prevalence (PP) of smoking abstinence at the end of the trial (week 12). Secondary outcomes included 4-week PP at week 12 and adherence, evaluated by proportion of days covered (PDC), to prescribed smoking cessation pharmacotherapies.

RESULTS: There were 1,017 randomized subjects. Among them, 1,002 subjects were included in the analysis, and 513 were randomized into EPC and 489 into UC. Baseline demographics, smoking history, and prescribed smoking cessation pharmacotherapies were comparable. Varenicline and nicotine replacement therapy (NRT) were most frequently prescribed for smoking cessation. In EPC, $46.0 \%$ received all 3 counseling sessions; $29.4 \%$ received 2 sessions; and $14.6 \%$ received 1 session. Overall, 353 subjects in EPC and 383 subjects in UC completed the week 12 assessment. In the analysis for 1-week PP of smoking abstinence at week 12, the percentage of abstainers in EPC was numerically higher than in UC $(42.3 \%$ vs. $38.2 \%$ ) with $\mathrm{OR}=1.24,95 \% \mathrm{Cl}=0.96-1.61$. It was not statistically significant. Adherence to prescription smoking cessation medication was significantly higher in EPC versus UC (49.7\% vs. $45.6 \%$; $P=0.033)$.

CONCLUSIONS: This study evaluated whether a telephone-based pharmacy care program, provided by pharmacists and designed to support attempted quitters, improved quitting and increased adherence over usual care.

The findings suggest that an enhanced program may benefit smokers by increasing prescription smoking cessation medication adherence. Future research should explore this program's effect on smokers who are compliant, based on insights on quitting provided by the post hoc analyses and limitations of the current study design.

J Manag Care Spec Pharm. 2016;22(12):1417-25

Copyright $\odot 2016$, Academy of Managed Care Pharmacy. All rights reserved.

\section{What is already known about this subject}

Smoking cessation counseling, coupled with pharmacotherapy, provides the best outcomes for smoking cessation.

Among health professionals, pharmacists are easily accessible to the public and can support discussions on smoking cessation directly with patients.

Smokers who have higher adherence to smoking cessation pharmacotherapy have better quitting outcomes.

\section{What this study adds}

This pragmatic randomized trial provides evidence that personalized interactive telephone counseling conducted by pharmacists supports adherence to prescribed smoking cessation therapies and quit attempts.

Pharmacists, who are trained in the pulmonary therapeutic area and in elements of motivational interviewing techniques, provided helpful support in subjects' quit attempts.

Telephone-based pharmacist counseling may be implemented by managed care organizations and policymakers to support smoking cessation efforts by their members.

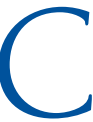
igarette smoking results in more than 480,000 premature deaths in the United States each year, and an additional 16 million people suffer from a serious illness caused by smoking. ${ }^{1}$ The prevalence of cigarette use is reported to be $26.2 \%$ in men and $15.4 \%$ in women. ${ }^{2}$ In the United States, the estimated economic cost of smoking is about $\$ 170$ billion in direct medical care for adults and more than $\$ 156$ billion in lost productivity. ${ }^{1}$ Smoking cessation has major and immediate health benefits and improves health-related quality of life..$^{3,4}$ For example, within 2 weeks to 3 months after quitting smoking, lung function and circulation improve; after 1 year, risk of coronary heart disease is half that of a current smoker., ${ }^{3,5,6}$

Smoking cessation counseling plays an integral role in smoking cessation treatment, either in conjunction with medication or alone. There are 7 first-line medications (5 nicotine and 2 non-nicotine) that have been confirmed to improve longterm quit rates. ${ }^{7}$ The 2008 Clinical Practice Guideline suggests that health care professionals should encourage all individuals making a quit attempt to use counseling and medication. Together, medication plus counseling demonstrate greater effectiveness than either alone. ${ }^{7}$ 
Health professionals, such as pharmacists, are in a unique position to assist smokers with quitting smoking. Specifically, pharmacists provide counseling on the effects of smoking and smoking cessation, the selection and appropriate use of a medication, the patient's coexistent medical conditions, and on the management of medication therapy. ${ }^{8-11}$ However, there are scarce data to evaluate pharmacists' telephone counseling. As the largest pharmacy benefits manager (PBM) in the United States with a centralized specialized pharmacist group, Express Scripts developed a telephone-based smoking cessation support program provided by specially trained pharmacists.

To examine the effect of a pharmacist-assisted intervention on a smoking cessation quit attempt, this pragmatic randomized trial evaluated the effect of pharmacist-provided telephone counseling calls on quitting smoking and adherence to pharmacotherapy in patients starting a pharmacotherapy-assisted quit attempt.

\section{Methods}

This study was designed as a prospective, randomized, pragmatic trial in a real-world setting to evaluate participating patients making a smoking cessation attempt using prescription pharmacotherapy. A pragmatic study design was selected because such studies address practical questions about the risks, benefits, and costs of an intervention as they would occur in routine clinical practice. ${ }^{12}$ Therefore, these studies may provide results about the specific population evaluated to providers, patients, payers, and other decision makers. ${ }^{13}$ Review and approval of the protocol were provided by Schulman Institutional Review Board before study initiation.

\section{Inclusion and Exclusion Criteria}

Inclusion criteria for subjects were as follows: Express Scripts members aged 21 years or older who were cigarette smokers and who had filled a new prescription for either varenicline (Chantix), bupropion HCL (Zyban), or nicotine replacement therapy (NRT, including transdermal patch, gum, inhalers, or nasal spray) within 5 days before study enrollment. A new prescription was a prescription for a smoking medication that the patient has not used in the previous 6 months. It was not a refill of a previous prescription. The Hierarchical Ingredient Code List (HICL) number is an identifier that represents an active ingredient and its specific therapeutic classification. For the purposes of this study, products were identified by the HICL number:

- Varenicline users were identified by prescriptions containing the HICL number 33766.

- Bupropion HCL users were identified by prescriptions containing the HICL number 01653 and the standard therapeutic classification code 9125. This requirement allowed differentiation between individuals taking bupropion HCL for smoking cessation and individuals taking Wellbutrin for depression.

- NRT was defined as nicotine-containing products identifiable by the HICL number 06249.

In addition, Express Scripts members must have had a minimum of 6 months previous continuous eligibility for pharmacy benefits; must have been capable of communicating in English on the telephone; and must have had telephone contact information in their pharmacy data.

Subjects were excluded who had received any prescription smoking cessation medications at the time of study enrollment or in the preceding 6 months as indicated in their pharmacy claims records or through self-reporting during the recruitment call. Upon enrollment into the study, subjects were expected to continuously use their new prescription smoking cessation medication until study completion. However, they were not excluded if they discontinued or switched to another smoking cessation medication or if they received any additional counseling support (i.e., literature pamphlets or online selfaccessed information). Additional exclusion criteria were any self-reported condition or circumstance that would prevent the subject from completing participation in the program for the full 3 months (i.e., scheduled medical procedures, travel, or inaccessibility by telephone); the subject's pharmacy data contained a "no contact" red flag; or the subject was covered by a nonparticipating employer group.

Phone numbers for subjects who passed prescreening for inclusion and exclusion criteria in the Express Scripts database were loaded into an autodialer and called up to 3 times. Those subjects who answered and were willing to receive a call from an Express Scripts Therapeutic Resource Center (TRC) pharmacy technician participated in a recruitment call to explain the study details, review the inclusion/exclusion criteria, and assess their willingness to participate. After confirmation of eligibility and informed consent, the subjects were randomized 1:1 to either the enhanced pharmacy care (EPC) group or the usual care (UC) group.

\section{Pharmacy Counseling Program}

Subjects in the EPC group received the Pharmacy Counseling Program (PCP), consisting of 3 counseling sessions via telephone at baseline, week 2, and week 5. These PCP sessions were provided by the Express Scripts TRC's specialist pharmacists who are uniquely trained in the pulmonary therapeutic area. The PCP program was designed in consultation with pharmacy practice researchers at Medco Health (now Express Scripts). Specifics of the program followed recommendations of the Clinical Practice Guideline ${ }^{7}$ as well as pharmacy standard practice. Counseling guides were provided during training of the specialist pharmacists to ensure consistency.

The objective of these outbound calls was to support the subject's quit attempt by maintaining motivation and commitment to the cessation process through personalized counseling sessions. Table 1 provides an overview of the PCP program, call length, and call topics. The sessions relied on a subject's individual pharmacy profile, incorporated elements of motivational interviewing techniques, focused on risks and consequences of smoking, explained the value of adherence to current medication and smoking cessation treatments, stressed the short-term and long-term benefits of smoking cessation, and described potential adverse events (AEs) of the prescribed smoking cessation therapy. Whenever possible, the same pharmacist made all 3 counseling calls to an individual subject to further 
TABLE 1 Pharmacist Counseling Program Design

\begin{tabular}{|c|c|c|c|}
\hline & Call 1 (Baseline) & Call 2 (Week 2) & Call 3 (Week 5) \\
\hline $\begin{array}{l}\text { Call frequency } \\
\text { (initial prescription fill=day 1) }\end{array}$ & Day $1-5$ & Day 17 (plus 7-day window) & Day 35 (plus 7-day window) \\
\hline Expected duration of call (minutes) & 30 & 15 & 15 \\
\hline Overview of call guide & $\begin{array}{l}\text { - Advise patient about recommen- } \\
\text { dations for quitting (inclusive of } \\
\text { counseling and medication, value } \\
\text { of adherence to current medication } \\
\text { regimen, and new smoking cessa- } \\
\text { tion therapeutics) } \\
\text { - Review medical and medication } \\
\text { history taken by technician } \\
\text { - Determine if patient has started } \\
\text { medication; provide medication } \\
\text { counseling } \\
\text { - Set quit date } \\
\text { - Develop/reinforce quit plan and } \\
\text { road blocks } \\
\text { - Set up next call }\end{array}$ & $\begin{array}{l}\text { - Assess smoking status } \\
\text { - Address any "slip-ups" } \\
\text { - Reinforce quitting commitment; } \\
\text { withdrawal symptoms are to be } \\
\text { expected } \\
\text { - Reassess confidence } \\
\text { - Patient-driven discussion on moti- } \\
\text { vation and health and lifestyle } \\
\text { benefits } \\
\text { - Review medication use } \\
\text { - Additional strategies to remain on } \\
\text { quit pathway }\end{array}$ & $\begin{array}{l}\text { - Similar elements to call \#2 with } \\
\text { addition of: } \\
\text { o This is the last call } \\
\text { o Stress the benefits of short- and } \\
\text { long-term smoking cessation on } \\
\text { patient's disease state(s) } \\
\text { o Review resources available to } \\
\text { support long-term quit plan } \\
\text { (i.e., helpful websites, applicable } \\
\text { phone numbers) }\end{array}$ \\
\hline
\end{tabular}

support the personalized interaction. Subjects in the UC group did not receive scheduled counseling. Subjects in both arms were allowed to make inbound calls to pharmacists as needed.

If the subject withdrew from the study and/or withdrew consent for disclosure of future information, no further evaluations were performed, and no additional data were collected. All interactions with subjects were conducted telephonically, inclusive of subjects' consent to participate in the study, pharmacist counseling, and data collection.

\section{Variables, Outcomes, and Statistical Analyses}

Baseline variables included demographics, medical and medication history, general health questions, smoking history, Fagerström Test for Nicotine Dependence (FTND), ${ }^{14,15}$ and motivation and confidence to quit questions. The total FTND score is based on 6 questions and is measured on a 0-10 scale. Interpretation of the total score is aided by considering 0-3 as corresponding to low nicotine dependence, 4- 6 as corresponding to moderate nicotine dependence, and 7-10 as corresponding to severe nicotine dependence..$^{15}$

Study outcomes were collected at week 12 through telephone contact, as well as through the Express Scripts prescription database. All subjects who did not withdraw from the study before week 12 were contacted for the week 12 data collection, regardless of whether the subject was compliant with the study procedure (i.e., counseling schedule in EPC group).

The primary outcome measure was the 1-week point prevalence (PP) of smoking abstinence at week 12. There were 2 possible answers to the question "Did you smoke any cigarettes (even a puff) in the past week?" where "yes" indicated nonabstinence (smoking) and "no" indicated abstinence. Subjects were asked to think back over the past 7 days to answer this question.

A secondary outcome measure assessed the 4-week PP of smoking abstinence at week 12 that was based on the response to the question "When was the last time you smoked any cigarette (even a puff)?". Subjects provided a response indicating the number of weeks since their last cigarette. Responses of 4 or higher were assigned "abstinence"; responses lower than 4 were "nonabstinence" (smoking); and unknown responses were recorded as missing. In both 1-week and 4-week abstinence analyses, missing responses were imputed as nonabstinence.

Likelihood ratio chi-square tests (SAS Proc Logistic) were used to assess the difference between EPC and UC groups, with odds ratios (ORs) calculated to identify the magnitude and direction of the differences. Two covariates, prescription therapy group (varenicline+bupropion and NRT + multiple + unknown Rx) and Baseline FTND, were included in the model to control for the potential impact they may have had on subjects' ability to quit smoking.

Secondary outcome measures also included an evaluation of the average proportion of days covered (PDC) by randomization group, irrespective of the types of smoking cessation medications, as a measure of medication adherence. ${ }^{16-18} \mathrm{PDC}$ is a function of medication availability over a period of time and was calculated by dividing the number of days that prescription smoking cessation medication was available by the required length of therapy prescribed for each medication, ${ }^{18}$ either 12 weeks (84 days) for varenicline and bupropion or 10 weeks (70 days) for NRT. This endpoint was available for subjects who took only 1 type of medication for the entire 12 -week study period; 10 subjects were excluded from each group because of multiple or unknown medications. PDC was analyzed by an analysis of covariance using PROC general linear model, including the same covariates as in the PP analyses.

Satisfaction with the PCP program in the EPC group was evaluated at week 12 with the question "Overall, how satisfied were you with the experience you had with the specialist pharmacist you spoke with today?". Responses were summarized 


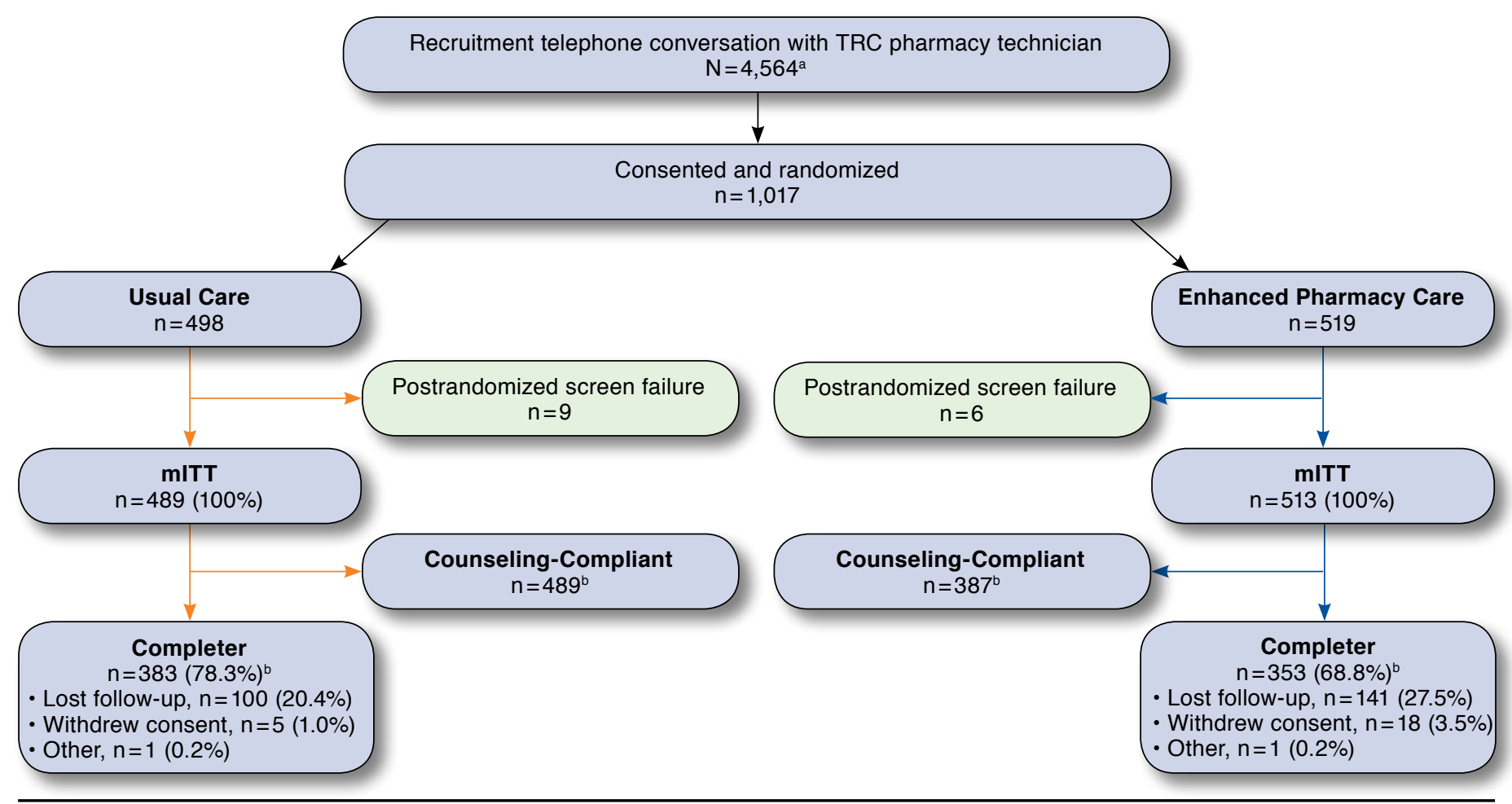

aThe counts were based on the study team's weekly recruitment reports and not from the final study database.

${ }^{b}$ Completer and Counseling-Compliant subsets were analyzed via post hoc analyses.

mITT = modified intent-to-treat; TRC = Therapeutic Resource Center.

descriptively and included the following: "extremely satisfied," "very satisfied," "somewhat satisfied," "not very satisfied," and "not at all satisfied."

To determine the sample size a priori, a l-week PP abstinence rate at week 12 was estimated as $45 \%$ for the EPC group, a $10 \%$ higher rate than the UC group. To identify at least a $10 \%$ higher abstinence rate among the EPC group than the UC group, the sample size was estimated to be 1,006 (503 per group) based on the following assumptions: 2-sided, 2-sample proportion test; $5 \%$ type I error; $90 \%$ power to detect a difference between the EPC and UC groups of at least 10\%; abstinence rate in the usual care group $=35 \%$; and an intent-to-treat (ITT) analysis. All statistical analyses were conducted using SAS, version 9.1 (SAS Institute, Cary, NC).

The prespecified analysis population was all randomized subjects (i.e., ITT population). However, during the study, a few subjects in both groups (15 total) were found to not meet inclusion/exclusion criteria after randomization because they were either "not a cigarette smoker" or "had taken a prescribed smoking cessation medicine in the past 6 months." These subjects were not enrolled and excluded before baseline data collection and did not participate in any further study procedures or outcomes data collection. Therefore, a modified ITT (mITT) population that included all randomized and enrolled subjects was used for all analyses. ${ }^{19}$

To better understand the magnitude of the effect of the counseling program among those who utilized it, post hoc sensitivity analyses were conducted on the following 2 subsets of the mITT population: (1) the Completer population included only subjects with nonmissing responses to the week 12 smoking abstinence questions, and (2) the Counseling-Compliant population included subjects who adhered to the intervention by completing at least 2 counseling calls.

AEs were collected during outbound calls from pharmacists to subjects ( 5 calls to EPC subjects and 2 calls to UC subjects). Subjects were asked the question "Have you experienced any side effects to the medication?". In addition, AEs that were reported on inbound calls to pharmacists were collected for both groups. All observed or volunteered AEs regardless of treatment group or suspected causal relationship to the prescription smoking cessation treatment were recorded by TRC pharmacists or their designees.

\section{Results}

As shown in Figure 1, 4,564 potential subjects had a recruitment conversation with a TRC technician, and 1,017 
TABLE 2 Baseline Characteristics: Modified Intent-to-Treat Subjects

\begin{tabular}{|c|c|c|c|c|}
\hline Demographics $^{a}$ & $\begin{array}{l}\text { Usual Care } \\
\mathrm{n}=489\end{array}$ & $\begin{array}{c}\text { Enhanced Pharmacy Care } \\
\mathrm{n}=513\end{array}$ & \multicolumn{2}{|c|}{$\begin{array}{c}\text { Total } \\
\mathrm{N}=1,002 \\
\end{array}$} \\
\hline Age (years), mean [SD] & $50.4 \quad[11.14]$ & $50.4 \quad[11.08]$ & 50.4 & [11.1] \\
\hline Sex: female & $292 \quad(59.7)$ & $296 \quad(57.7)$ & 588 & $(58.7)$ \\
\hline Race: white & $438 \quad(89.6)$ & $435 \quad(84.8)$ & 873 & $(87.1)$ \\
\hline BMI $\left(\mathrm{kg} / \mathrm{mg}^{2}\right)$, mean [SD] & $29.03 \quad[6.12]$ & $28.43 \quad[5.95]$ & 28.72 & {$[6.04]$} \\
\hline \multicolumn{5}{|l|}{ Smoking cessation prescription medications } \\
\hline Varenicline & $422 \quad(86.3)$ & $429 \quad(83.6)$ & 851 & $(84.9)$ \\
\hline NRT & $50 \quad(10.2)$ & $65 \quad(12.7)$ & 115 & $(11.5)$ \\
\hline Bupropion & $(1.4)$ & $(1.8)$ & 16 & $(1.6)$ \\
\hline Multiple Rx & $(0.8)$ & $(1.2)$ & 10 & $(1.0)$ \\
\hline Unknown Rx & $(1.2)$ & $(0.8)$ & 10 & $(1.0)$ \\
\hline \multicolumn{5}{|l|}{ Medical history } \\
\hline \multicolumn{5}{|l|}{ General health question "Your health is...." } \\
\hline "Good," "Very Good," or "Excellent" & $387 \quad(79.1)$ & $386 \quad(75.2)$ & 773 & $(77.1)$ \\
\hline \multicolumn{5}{|l|}{ Health conditions } \\
\hline At least 1 abnormal condition & $418 \quad(85.5)$ & $440 \quad(85.8)$ & 858 & $(85.6)$ \\
\hline Cardiovascular disease & $202 \quad(41.3)$ & $230 \quad(44.8)$ & 432 & $(43.1)$ \\
\hline Chronic pain & $158 \quad(32.3)$ & $161 \quad(31.4)$ & 319 & $(31.8)$ \\
\hline Depression & $116 \quad(23.7)$ & $140 \quad(27.3)$ & 256 & $(25.5)$ \\
\hline GI problems & $114 \quad(23.3)$ & $130 \quad(25.3)$ & 244 & $(24.4)$ \\
\hline Pulmonary problems (other than cancer) & $72 \quad(14.7)$ & $97 \quad(18.9)$ & 169 & $(16.9)$ \\
\hline Diabetes & $72 \quad(14.7)$ & $84 \quad(16.4)$ & 156 & $(15.6)$ \\
\hline Cancer & $52 \quad(10.6)$ & $(10.3)$ & 105 & $(10.5)$ \\
\hline \multicolumn{5}{|l|}{ Smoking history } \\
\hline FTND score, mean [SD] & {$[2.22]$} & $4.6 \quad[2.21]$ & 4.4 & {$[2.21]$} \\
\hline Cigarettes smoked per day, mean [SD] & {$[9.15]$} & $18.3 \quad[8.77]$ & 18.4 & {$[8.95]$} \\
\hline \multicolumn{5}{|l|}{ Last time quit smoking for at least 1 day } \\
\hline$\leq 12$ months ago & $(37.4)$ & $195 \quad(38.0)$ & 378 & $(37.7)$ \\
\hline$\geq 12$ months ago & $275 \quad(56.2)$ & $289 \quad(56.3)$ & 564 & $(56.3)$ \\
\hline Never & $(6.3)$ & $(5.7)$ & 60 & $(6.0)$ \\
\hline \multicolumn{5}{|l|}{ Methods used in the last 12 months } \\
\hline Cold turkey & $150 \quad(30.7)$ & $(27.5)$ & 291 & $(29.0)$ \\
\hline OTC NRT & $74 \quad(15.1)$ & $(16.2)$ & 157 & $(15.7)$ \\
\hline Prescription smoking cessation pharmacotherapy & $(17.6)$ & $(17.7)$ & 177 & $(17.7)$ \\
\hline Baseline $^{\mathrm{b}}$ motivation to quit, meanc [SD] & [1.59] & [1.65] & 8.8 & [1.62] \\
\hline 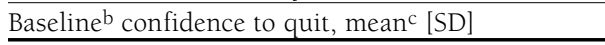 & $8.2 \quad[1.76]$ & {$[1.95]$} & 8.1 & [1.86] \\
\hline
\end{tabular}

Note: $n(\%)$ shown unless otherwise noted.

${ }^{a}$ Age and sex were obtained from pharmacy claims database. All other characteristics were subject-reported.

${ }^{b}$ Baseline $=$ at enrollment.

cScored on scale of 1-10, where 10 is high.

$B M I=$ body mass index; FTND = Fagerstrom Test for Nicotine Dependence; GI=gastrointestinal; NRT=nicotine replacement therapy; OTC=over-the-counter;

$R x=$ prescription; $S D=$ standard deviation.

subjects consented to participate and were randomized $(1,017 / 4,564=22.3 \%)$. Of those, 15 subjects were excluded after randomization without any data collection because they did not meet all inclusion criteria. Therefore, 1,002 subjects were enrolled and included in the study analyses as the mITT population: 513 in the EPC group and 489 in the UC group. Overall, $736(73.5 \%)$ subjects-353 (48.0\%) in the EPC group and 383 (52.0\%) in the UC group-completed the study with both baseline and week 12 assessment data regardless of compliance to the smoking cessation medications or counseling schedules.
The distributions of subjects among prescription smoking cessation medications were comparable across groups (Table 2): $83.6 \%$ and $86.3 \%$ of subjects took varenicline in the EPC and UC groups, respectively; $12.7 \%$ and $10.2 \%$ took NRT; $1.8 \%$ and $1.4 \%$ took bupropion; and $2.0 \%$ and $2.0 \%$ took multiple/ unknown medications over the 12-week study. Baseline characteristics of study subjects in the EPC and UC groups were comparable (Table 2).

The mITT study population included $40.3 \%$ males and $58.7 \%$ females, had a mean age of 50.4 years (standard 


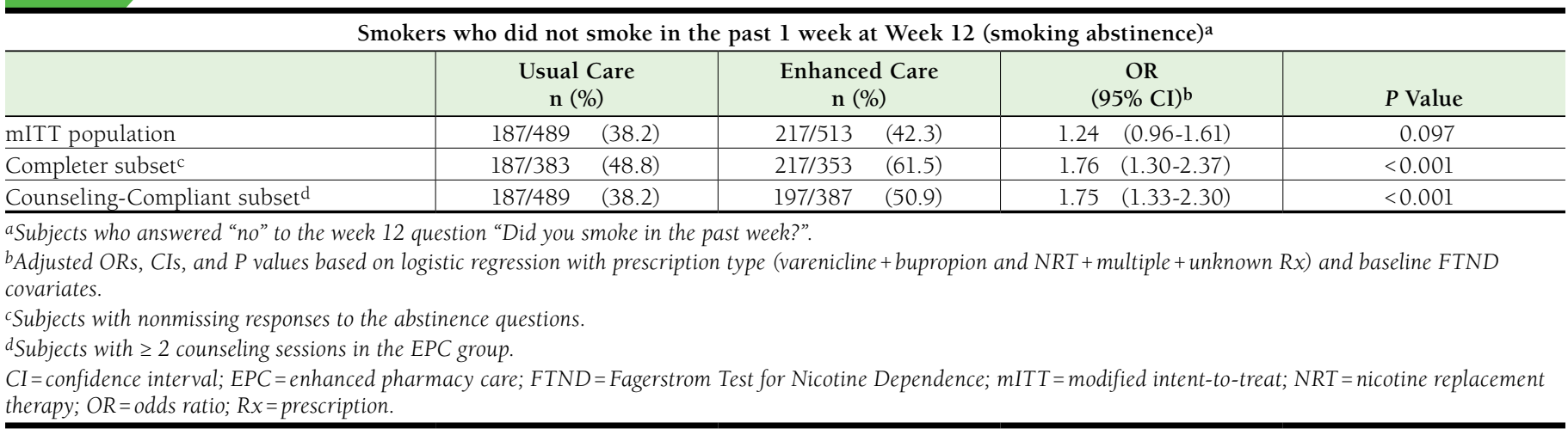

deviation $[S D]=11.10$ ), and $87.1 \%$ described their race as white. Most subjects (85.6\%) reported at least 1 medical condition during the recruitment calls. The most prevalent self-reported medical conditions were cardiovascular disease, chronic pain, depression, and gastrointestinal disorders. Subjects smoked an average of 18 cigarettes a day according to their responses to recruitment questions and had a mean FTND baseline score of $4.4(\mathrm{SD}=2.21)$ out of a maximum of 10 . About $38 \%$ of subjects reported a quit attempt within the previous 12 months. However, less than $8 \%$ of subjects indicated that they were counseled by a health care professional during the previous 12 months, and almost none of that counseling was provided by pharmacists. On a scale of $1-10$, with 10 being completely motivated/confident, the EPC average scores were 8.7 and 8.0, and the UC average scores were 8.8 and 8.2 for motivation and confidence at baseline, respectively (Table 2).

Among 513 subjects in the EPC group, $75.4 \%$ of subjects had at least 2 contacts by week 12 (46.0\% received all 3 counseling sessions; $29.4 \%$ received 2 sessions). About $10 \%$ of the population did not participate in any counseling sessions.

At the week 12 assessment, in response to the question "Did you smoke any cigarettes (even a puff) in the past week?", 160 of the 513 subjects in the EPC group (31.2\%) and 106 of the 489 subjects in the UC group (21.7\%) had a missing response. For the primary efficacy endpoint of 1-week PP of smoking abstinence, when missing responses were imputed as nonabstinence, $42.3 \%$ of subjects in the EPC group and $38.2 \%$ in the UC group were abstainers. Although the result was numerically higher in the EPC group with an OR of 1.24 (95\% confidence interval $[\mathrm{CI}]=0.96-1.61$ ) adjusted for prescription group and baseline FTND, it was not statistically significant $(P=0.097$; Table 3). The 4-week abstinence result was similar (34.1\% and $32.3 \%$ in EPC and UC, respectively; adjusted OR $=1.14,95 \%$ $\mathrm{CI}=0.87-1.49 ; \mathrm{P}=0.344)$.

In PDC analysis, when controlling for prescription and baseline FTND score, the EPC group had a significantly higher PDC compared with the UC group (49.7\% vs. $45.6 \%$; $P=0.033$ ). This indicated that the EPC group had significantly higher adherence to the prescribed smoking cessation therapy than the UC group. When the PDC data by prescribed smoking cessation medications were investigated, the PDCs were numerically higher in the EPC subgroups than in the UC subgroups for varenicline (50.4\% vs. $46.5 \%$ ) and NRT subjects $(45.9 \%$ vs. $37.9 \%$ ). Statistical testing was not done for these subgroup comparisons.

The post hoc Completer analysis (Table 3) was based on the 736 EPC and UC subjects who responded to the week 12 smoking questions. Baseline characteristics of the post hoc analyses were comparable between the corresponding EPC subgroups and UC subgroups, which can be found in the Appendix (available in online article). Adjusting for the same covariates, the EPC group had significantly higher 1-week abstinence than the UC group (61.5\% vs 48.8\%; adjusted OR $=1.76$, 95\% CI $=1.30$ 2.37; $P<0.001)$. Similarly, 4 -week abstinence was significantly higher in the EPC group (49.6\% vs. $41.3 \%$, adjusted $\mathrm{OR}=1.46$, 95\% CI $=1.08-1.97 ; P=0.013)$.

The post-hoc Counseling-Compliant analysis (Table 3) showed that 1-week abstinence was significantly higher in the EPC group than in the UC group after adjusting for prescription and baseline FTND (50.9\% vs. 38.2\%; adjusted $\mathrm{OR}=1.75,95 \% \mathrm{CI}=1.33-2.30 ; \mathrm{P}<0.001)$. The 4 -week abstinence rate was also significantly higher in the EPC group (41.6\% vs. $32.3 \%$, adjusted $\mathrm{OR}=1.56,95 \% \mathrm{CI}=1.18-2.06$; $P=0.002)$.

In response to the question on satisfaction with the PCP program, 93.7\% (329/351 EPC subjects responding) were extremely or very satisfied with the experience. Other answers included "somewhat satisfied," "not very satisfied," and "not at all satisfied" (22 subjects responding).

Nausea and abnormal dreams were the most frequently reported AEs in subjects taking varenicline in both EPC and UC groups (Table 4). For subjects taking NRT, the most frequently reported $\mathrm{AE}$ was abnormal dreams.

\section{Discussion}

Tobacco use is the single most preventable cause of death and disease in the United States, and the health benefits that result from quitting are substantial. ${ }^{1,3-6}$ Previously published 
TABLE 4 Adverse Events as Reported by Subjects

Enhanced

Usual Care

(Up to 2

Scheduled Calls)

Pharmacy Care

(Up to 5

Scheduled Calls)

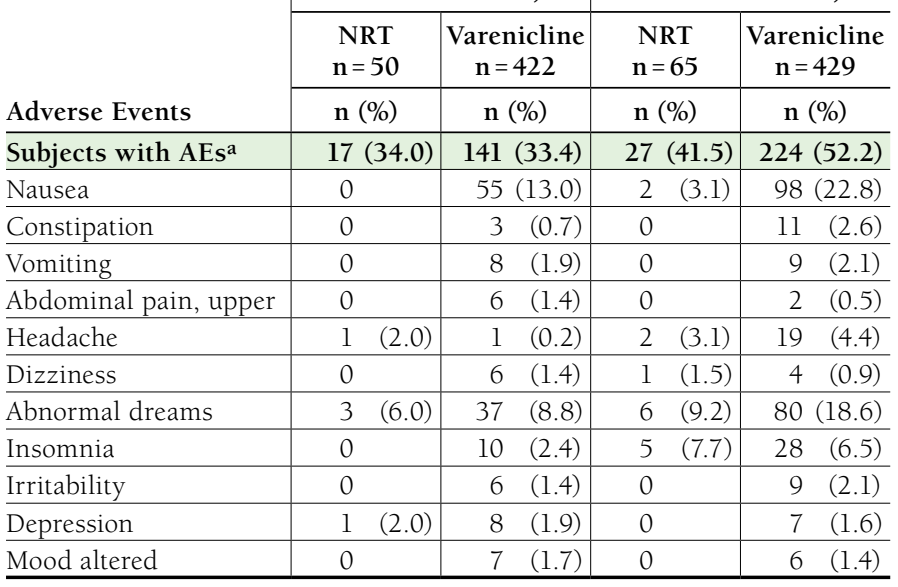

Note: Subjects are counted only once in each preferred term category.

${ }^{a}$ AEs with frequency $\geq 1 \%$ in UC varenicline subjects and/or $\geq 2 \%$ in EPC

varenicline subjects.

$A E=$ adverse event $E P C=$ enhanced pharmacy care; $N R T=$ nicotine replacement

therapy; $U C=$ usual care.

pragmatic studies have demonstrated that health care providers, especially pharmacists, can help motivate smokers to quit smoking. ${ }^{11}$ This study was designed to evaluate the efficacy of a pharmacist-provided telephone support program through a PBM by assessing the effect of telephone support on a sample of patients newly initiating a prescription for smoking cessation pharmacotherapy. The results of the main analysis were inconclusive in demonstrating a significant benefit. However, 2 post hoc analyses provided some insights that may be useful in assessing whether to institute such a program.

This pragmatic study has multiple strengths. First, the study was conducted using 2-5 telephone calls scheduled at subjects' convenience, so no pharmacy or clinic visit was required; therefore, disruption to treatment routines was minimal. Second, the large sample size, short list of subject selection criteria, and randomized design ensured the representativeness of the results, minimized the bias of subject selection, and strengthened the objectiveness of the outcome analyses. Third, the study was conducted by a group of specialist pharmacists who had been trained for the PCP program to ensure the consistency and accuracy of delivery of the intervention (counseling) and collection of data.

In addition, the post hoc Counseling-Compliant subset, where subjects completed at least 2 counseling calls, provided key insight into understanding the population that optimally used the pharmacist-provided telephone support program. smoking cessation is a personal choice, and the type of support system that smokers find helpful may vary between individuals. The Counseling-Compliant subset was representative of those subjects who opted to sufficiently use the program. The abstinence rate in the Counseling-Compliant analysis was statistically significantly higher in the EPC group than that in the UC group. This result indicates that receiving at least 2 counseling calls may have been useful in supporting a successful quit attempt among those smokers who took advantage of the program. On the other hand, by continuing the intervention, there might be self-selection of the subjects more willing to take an active part to quit smoking, although the similarity of baseline characteristics between the 2 groups was reassuring in this regard.

\section{Limitations}

This study had some limitations that must be acknowledged. The pragmatic design may have led to some difficulties in data collection, resulting in an imbalance of missing data between the 2 groups. In the primary analysis, the calculations of the abstinence rates were based on a conservative approach, which imputed the missing data as nonabstinence. In this way, the abstinence rates were likely underestimated. In addition, the imbalance in missing responses between the 2 groups potentially introduced bias into the between-group comparisons. It is worth noting that the adherence analysis in the mITT population found that EPC subjects had a significantly higher adjusted mean PDC of prescribed pharmacotherapies, compared with UC subjects. Previous research has indicated that smokers who are more adherent to smoking cessation pharmacotherapy have better quit rates. ${ }^{20}$ This dichotomy illustrates the importance of missing data handling. Such considerations should influence the design and analysis of future studies where possible missing data patterns are considered a priori rather than post hoc.

While the post hoc analyses were used to minimize the effect of missing responses and provided some evidence that subjects completing at least 2 sessions had higher self-reported abstinence, caution must be used in interpreting the results because of the lack of randomization in these subgroup analyses. The usefulness of these post hoc analyses are to point toward an avenue of future research where the limitations of the current study design can be addressed.

Additional limitations are worth mentioning. The design used a short follow-up period with a self-reported, unverified abstinence endpoint. Additionally, few (15) randomized subjects were excluded from the study analyses, which should not have affected data interpretation considering the overall large sample size of the study. Given the nature of telephone-based surveys, generalizability of the results was restricted to those who had access to a telephone and a desire to participate in the study, but the responses to the survey questionnaires cannot be verified. Finally, subjects in the EPC group had a higher rate of AEs compared with subjects in the UC group, possibly because subjects in the EPC group had the opportunity of repeated contacts (and, thus, repeated opportunities for reporting events) with the specialist pharmacist. 


\section{Conclusions}

Evidence-based approaches to smoking cessation are important to maximize the success of smokers who reach their goals of cessation and of payers and policymakers who implement effective programs into practice. The results of this study give useful information about the pharmacist-provided telephone counseling program. Managed care organizations and policymakers might consider adding telephone-based pharmacist counseling to their services to support smoking cessation efforts by their members.

\section{Authors}

JASON GONG, MD, and ZHANNA JUMADILOVA, MD, MBA, Clinical Sciences, Pfizer, New York, New York. KELLY H. ZOU, $\mathrm{PhD}$, and DAVID LAWRENCE, PhD, Global Innovative Pharma Business-Statistics, Pfizer, New York, New York. CHRISTINE L. BAKER, JD, MPH, Global Health and Value, and MARIANNA BRUNO, PharmD, MPH, Medical Affairs North America, Pfizer, New York, New York. BETH WILSON, MS, MPH, and CYNTHIA EWEL, PhD, United BioSource Corporation, Blue Bell, Pennsylvania. AUTHOR CORRESPONDENCE: Cynthia Ewel, PhD, United BioSource Corporation, 920 Harvest Dr., Blue Bell, PA 19422. Tel.: 240.271.1666; E-mail: cynthia.ewel@ubc.com.

\section{DISCLOSURES}

This study was sponsored by Pfizer. Gong, Baker, Zou, Bruno, Jumadilova, and Lawrence are employees and stockholders of Pfizer. Wilson and Ewel are employees of United BioSource Corporation, which received funding from Pfizer for conducting this study and for the development of this manuscript.

Study concept and design were contributed by Gong, Bruno, and Ewel, with assistance from Jumadilova, Lawrence, and Zou. Gong, Jumadilova, Lawrence, and Ewel collected the data. Data interpretation was performed by Baker, Zou, and Wilson, assisted by Gong, Lawrence, and Ewel. The manuscript was written by Baker, Ewel, and Gong, with assistance from the other authors, and revised by Baker, Wilson, Zou, and Gong, with assistance from Bruno and Jumadilova.

\section{ACKNOWLEDGMENTS}

This manuscript was edited by Jyoti Nandi, PhD, and Deborah Z. O'Donnell, BA, employees of United BioSource Corporation, and funded by Pfizer.

\section{REFERENCES}

1. U.S. Department of Health and Human Services. The Health Consequences of Smoking: 50 Years of Progress. A Report of the Surgeon General. Atlanta, GA: U.S. Department of Health and Human Services, Centers for Disease Control and Prevention, National Center for Chronic Disease Prevention and Health Promotion, Office on Smoking and Health; 2014. Printed with corrections, January 2014.

2. Agaku I, King B, Husten C, et al. Tobacco product use among adultsUnited States, 2012-2013. MMWR Morb Mortal Wkly Rep. 2014;63(25):542-47.

3. U.S. Department of Health and Human Services. The health benefits of smoking cessation: a report of the Surgeon General. DHHS Publication No. (CDC) 90-8416. U.S. Department of Health and Human Services, Public Health Service, Centers for Disease, Center for Chronic Disease Prevention and Health Promotion, Office on Smoking and Health. 1990. Available at: https://profiles.nlm.nih.gov/NN/B/B/c/T/. Accessed October 7, 2016.

4. Tomioka H, Sekiya R, Nishio C, Ishimoto G. Impact of smoking cessation therapy on health-related quality of life. BMJ Open Respir Res. 2014;1(1):e000047.

5. U.S. Department of Health and Human Services. A report of the Surgeon General: how tobacco smoke causes disease: what it means to you. U.S. Department of Health and Human Services, Centers for Disease Control and Prevention, National Center for Chronic Disease Prevention and Health Promotion, Office on Smoking and Health. 2010. Available at: http://www.cdc. gov/tobacco/data_statistics/sgr/2010/consumer_booklet/pdfs/consumer.pdf. Accessed October 7, 2016.

6. American Cancer Society. Benefits of quitting smoking over time. Revised September 9, 2016. Available at: http://www.cancer.org/healthy/stayawayfromtobacco/benefits-of-quitting-smoking-over-time. Accessed October 19, 2016.

7. Fiore MC, Jaén CR, Baker TB, et al. Treating tobacco use and dependence: 2008 update. Clinical Practice Guideline. U.S. Department of Health and Human Services, Public Health Service. May 2008. Available at: http://bphc. hrsa.gov/buckets/treatingtobacco.pdf. Accessed October 7, 2016.

8. Shen X, Bachyrycz A, Anderson JR, Tinker D, Raisch DW. Quitting patterns and predictors of success among participants in a tobacco cessation program provided by pharmacists in New Mexico. J Manag Care Spec Pharm. 2014;20(6):579-87. Available at: http://www.jmcp.org/doi/10.18553/ jmcp.2014.20.6.579.

9. Patwardhan PD, Chewning BA. Tobacco users' perceptions of a brief tobacco cessation intervention in community pharmacies. J Am Pharm Assoc (2003). 2010;50(5):568-74.

10. Munarini E, Marabelli C, Marmotti A, et al. Antismoking centers in Milan's communal pharmacies: analysis of the 2010-2011 campaign. Tumori. 2013;99(5):578-82.

11. Bock BC, Hudmon KS, Christian J, Graham AL, Bock FR. A tailored intervention to support pharmacy-based counseling for smoking cessation. Nicotine Tob Res. 2010;12(3):217-25.

12. Roland M, Torgerson DJ. What are pragmatic trials? BMJ. 1998;316(7127):285.

13. Peikes D, Geonnotti K, Wang W. Using pragmatic clinical trials to test the effectiveness of patient-centered medical home models in real-world settings. AHRQ Publication No. 13-0030-EF. February 2013. Agency for Healthcare Research and Quality. Rockville, MD. Available at: https://pcmh. ahrq.gov/sites/default/files/attachments/UsingPragmatic_032513comp.pdf. Accessed October 7, 2016.

14. Heatherton TF, Kozlowski LT, Frecker RC, Fagerström KO. The Fagerström Test for Nicotine Dependence: a revision of the Fagerstrom Tolerance Questionnaire. Br J Addict. 1991;86(9):1119-27.

15. Fagerström Kl, Russ C, Yu CR, Yunis C, Foulds J. The Fagerström Test for Nicotine Dependence as a predictor of smoking abstinence: a pooled analysis of varenicline clinical trial data. Nicotine Tob Res. 2012;14(12):1467-73.

16. Cramer JA, Roy A, Burrell A, et al. Medication compliance and persistence: terminology and definitions. Value Health. 2008;11(1):44-47.

17. International Society of Pharmacoeconomics and Outcomes Research (ISPOR). Medication Adherence and Persistence Special Interest Group. December 1, 2015. Available at: http://www.ispor.org/sigs/medication.asp. Accessed October 7, 2016.

18. Siu AL. Behavioral and pharmacotherapy interventions for tobacco smoking cessation in adults, including pregnant women: U.S. Preventive Services Task Force recommendation statement. Ann Intern Med. 2015;163(8):622-34.

19. Abraha I, Montedori A. Modified intention to treat reporting in randomised controlled trials: systematic review. BMJ. 2010;340:c2697.

20. Lieberman JN, Lichtenfeld MJ, Galaznik A, et al. Adherence to varenicline and associated smoking cessation in a community-based patient setting. J Manag Care Pharm. 2013;19(2):125-31. Available at: http://www.jmcp. org/doi/10.18553/jmcp.2013.19.2.125. 


\begin{tabular}{|c|c|c|c|c|}
\hline & $\begin{array}{c}\text { Completer } \\
\text { Usual Care } \\
\mathbf{n}=383\end{array}$ & $\begin{array}{c}\text { Completer } \\
\text { Enhanced Pharmacy } \\
\text { Care } \\
\mathbf{n}=353\end{array}$ & $\begin{array}{c}\text { Counseling-Compliant } \\
\text { Usual Care } \\
\mathbf{n}=489\end{array}$ & $\begin{array}{c}\text { Counseling-Compliant } \\
\text { Enhanced Pharmacy } \\
\text { Care } \\
\mathbf{n}=387\end{array}$ \\
\hline \multicolumn{5}{|l|}{ Demographics a } \\
\hline Age (years), mean [SD] & $51.2[10.98]$ & 51.2 [11.33] & $50.4[11.14]$ & 51.0 [11.03] \\
\hline Sex: Female & $228 \quad(59.5)$ & $206 \quad(58.4)$ & $292 \quad(59.7)$ & $217 \quad(56.1)$ \\
\hline Race: White & $342(89.3)^{b}$ & $298 \quad(84.4)$ & $438(89.6)^{b}$ & $326 \quad(84.2)$ \\
\hline BMI $\left(\mathrm{kg} / \mathrm{mg}^{2}\right)$, mean $[\mathrm{SD}]$ & $29.0 \quad[6.07]$ & $28.4 \quad[5.91]$ & $29.0 \quad[6.12]$ & $28.4 \quad[5.95]$ \\
\hline \multicolumn{5}{|l|}{ Smoking cessation prescription medications } \\
\hline Varenicline & $332 \quad(86.7)$ & $298 \quad(84.4)$ & $422 \quad(86.3)$ & $325 \quad(84.0)$ \\
\hline NRT & $(9.7)$ & $43 \quad(12.2)$ & $50 \quad(10.2)$ & $48 \quad(12.4)$ \\
\hline Bupropion & $(1.3)$ & $(1.4)$ & $(1.4)$ & $(1.6)$ \\
\hline Multiple Rx & $(1.0)$ & (1.4) & $(0.8)$ & $(1.3)$ \\
\hline Unknown Rx & $(1.3)$ & $(0.6)$ & $(1.2)$ & $(0.8)$ \\
\hline \multicolumn{5}{|l|}{ Medical history } \\
\hline \multicolumn{5}{|l|}{ General health question "Your health is..." } \\
\hline "Good," "very good," or "excellent" & $304 \quad(79.4)$ & $265 \quad(75.1)$ & $387 \quad(79.1)$ & $292 \quad(75.5)$ \\
\hline \multicolumn{5}{|l|}{ Health conditions } \\
\hline At least 1 abnormal condition & $332 \quad(86.7)$ & $304 \quad(86.1)$ & $418 \quad(85.5)$ & $329 \quad(85.0)$ \\
\hline Cardiovascular disease & $161 \quad(42.0)$ & $169 \quad(47.9)$ & $202 \quad(41.3)$ & $177 \quad(45.7)$ \\
\hline Chronic pain & $124 \quad(32.4)$ & $109 \quad(30.9)$ & $158 \quad(32.3)$ & $120 \quad(31.0)$ \\
\hline Depression & $93 \quad(24.3)$ & $100 \quad(28.3)$ & $116 \quad(23.7)$ & $113 \quad(29.2)$ \\
\hline GI problems & $94 \quad(24.5)$ & $99 \quad(28.0)$ & $114 \quad(23.3)$ & $104 \quad(26.9)$ \\
\hline Pulmonary problems (other than cancer) & $58 \quad(15.1)$ & $70 \quad(19.8)$ & $72 \quad(14.7)$ & $75 \quad(19.4)$ \\
\hline Diabetes & $59 \quad(15.4)$ & $61 \quad(17.3)$ & $72 \quad(14.7)$ & $66 \quad(17.1)$ \\
\hline Cancer & $42 \quad(11.0)$ & $42 \quad(11.9)$ & $52 \quad(10.6)$ & $42 \quad(10.9)$ \\
\hline \multicolumn{5}{|l|}{ Smoking history } \\
\hline FTND score, mean [SD] & $4.3 \quad[2.25]$ & $4.4 \quad[2.22]$ & $\begin{array}{ll}4.3 & {[2.22]} \\
\end{array}$ & $4.5 \quad[2.22]$ \\
\hline Cigarettes smoked per day, mean [SD] & $18.3 \quad[9.34]$ & 17.8 [8.97] & $18.4 \quad[9.15]$ & $18.3[9.04]$ \\
\hline \multicolumn{5}{|l|}{ Last time quit smoking for at least 1 day } \\
\hline$\leq 12$ months ago & $141 \quad(36.8)$ & $138 \quad(39.1)$ & $183 \quad(37.4)$ & $149 \quad(38.5)$ \\
\hline$\geq 12$ months ago & $219 \quad(57.2)$ & $198 \quad(56.1)$ & $275 \quad(56.2)$ & $216 \quad(55.8)$ \\
\hline Never & $23 \quad(6.0)$ & $(4.8)$ & $(6.3)$ & $(5.7)$ \\
\hline \multicolumn{5}{|l|}{ Methods used in the last 12 months } \\
\hline Cold turkey & $119 \quad(31.1)$ & $99 \quad(28.0)$ & $150 \quad(30.7)$ & $107 \quad(27.6)$ \\
\hline OTC NRT & $58 \quad(15.1)$ & $53 \quad(15.0)$ & $74 \quad(15.1)$ & $63 \quad(16.3)$ \\
\hline Prescription SC pharmacotherapy & $64 \quad(16.7)$ & $73 \quad(20.7)$ & $86 \quad(17.6)$ & $70 \quad(18.1)$ \\
\hline Baseline $^{\mathrm{c}}$ motivation to quit, mean ${ }^{\mathrm{d}}$ [SD] & $8.8 \quad[1.61]$ & $8.7 \quad[1.65]$ & $8.8 \quad[1.59]$ & $8.8 \quad[1.62]$ \\
\hline Baselinec confidence to quit, meand $[S D]$ & $8.2 \quad[1.82]$ & $8.1 \quad[1.94]$ & $\begin{array}{ll}8.2 & {[1.76]} \\
\end{array}$ & $8.1 \quad[1.88]$ \\
\hline \multicolumn{5}{|c|}{ 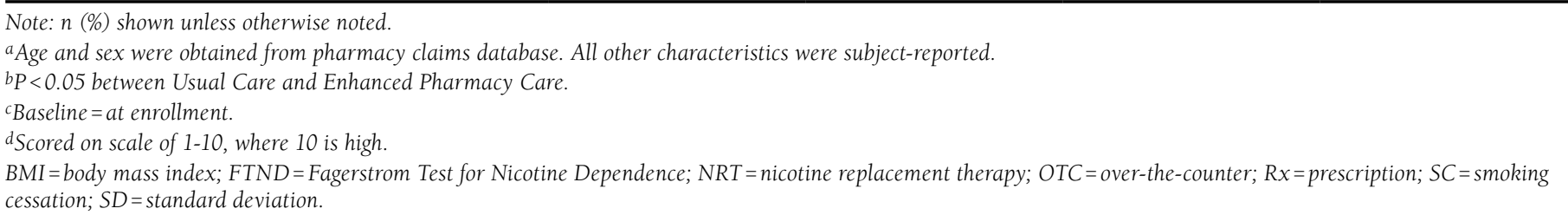 } \\
\hline
\end{tabular}

